\title{
Observations of ionospheric heating during the passage of solar coronal hole fast streams
}

\author{
J. J. Sojka, ${ }^{1}$ R. L. McPherron, ${ }^{2}$ A. P. van Eyken, ${ }^{3}$ M. J. Nicolls, ${ }^{3}$ C. J. Heinselman, ${ }^{3}$ \\ and J. D. Kelly ${ }^{3}$
}

Received 5 May 2009; revised 1 September 2009; accepted 10 September 2009; published 13 October 2009.

[1] Using ionospheric temperature measurements made continuously by the Poker Flat Incoherent Scatter Radar (PFISR) and EISCAT Svalbard Radar (ESR) during the International Polar Year (IPY), we provide evidence for directly driven ionospheric heating associated with the solar wind corotating interaction region (CIR). Both ESR and PFISR operated almost continuously during the IPY, which began on 1 March 2007. During this period 55 CIR events occurred and when ISR observations were available during these events ionospheric heating was observed. This study is the first comprehensive observation of ionospheric heating by CIRs demonstrated through case study comparisons and statistically over the 1-year IPY period. These multiple-day heating events are present in both the auroral and polar regions. The quantitative one-to-one correlation between ACE-CIR observations and ISRionosphere observations leads to a database that will enable the ionospheric heating efficiency of CIR events to be determined. Citation: Sojka, J. J., R. L. McPherron, A. P. van Eyken, M. J. Nicolls, C. J. Heinselman, and J. D. Kelly (2009), Observations of ionospheric heating during the passage of solar coronal hole fast streams, Geophys. Res. Lett., 36, L19105, doi:10.1029/2009GL039064.

\section{Introduction}

[2] Two recent discoveries of thermospheric periodicities in power radiated by carbon dioxide and nitric oxide [Mlynczak et al., 2008] and of increased thermospheric neutral mass density [Lei et al., 2008; Thayer et al., 2008] have shown very strong correlations with solar wind CIRs. Mlynczak et al. [2008] analyzed infrared observations from the SABER instrument on NASA TIMED satellite over the 100 to $200 \mathrm{~km}$ altitude region in the thermosphere. Their spectral analysis found a nine-day periodicity in their data set spanning 2002 through 2006 . The same periodicity is found in the $\mathrm{Kp}$ and Ap geomagnetic indices, but not in the solar EUV. They connected this nine-day periodicity to that found by Temmer et al. [2007] in the occurrence of coronal hole features on the sun in the 1998-2006 time frame. The second discovery by Lei et al. [2008] using measurements of non-gravitational accelerations (changes in neutral mass

\footnotetext{
${ }^{1}$ Center for Atmospheric and Space Sciences, Utah State University, Logan, Utah, USA.

${ }^{2}$ Institute of Geophysics and Planetary Physics, University of California, Los Angeles, California, USA.

${ }^{3}$ Center for Geospace Studies, SRI International, Menlo Park, California, USA.
}

Copyright 2009 by the American Geophysical Union. 0094-8276/09/2009GL039064\$05.00 density) on the CHAMP satellite also found a nine-day periodicity. Thayer et al. [2008] extended this analysis to show that periodicities of $4-5,6-7$, and 9-11 days were present. Recently observations by the GUVI instruments on the TIMED satellites have observed the CIR periodicity in the thermosphere and ionosphere [Crowley et al., 2008].

[3] CIR events in the solar wind have a well-established connection to solar coronal holes and are associated with high-speed flows. The geospace response to the CIR is very different from that of superstorms, which are driven by coronal mass ejections (CMEs). Borovsky and Denton [2006] describe 21 ways in which these two heliospheric phenomena are different. CIR events do not produce large geomagnetic storms as their minimum median Dst rarely gets below -30 nT [McPherron and Siscoe, 2004; McPherron and Weygand, 2006]. Hence, they are not viewed as a major space weather phenomenon. However, the two recent thermospheric discoveries demonstrate this classification to be an oversight and raise the question of how effectively CIR events heat the thermosphere.

[4] In this study, we present evidence that connects the heating in the thermosphere and ionosphere to the CIR using ionospheric polar cap and auroral observations made continuously through the IPY, 1 March 2007 to 29 February 2008, by the EISCAT Svalbard Radar (ESR) and the Poker Flat Incoherent Scatter Radar (PFISR). The continuous operations of these ISRs provide data sets that contain the ionospheric response to every CIR event observed by the Advanced Composition Explorer (ACE) satellite, which monitors solar wind conditions. Our study finds the events on a case-by-case basis and does not require a statistical analysis to establish the link. Using the ionospheric ion temperature measurements, we demonstrate the CIR ionospheric relationship quantitatively.

\section{IPY Campaign}

[5] An international campaign was organized in conjunction with the IPY to continuously observe the high latitude and polar ionosphere. This ambitious objective required running ISRs continuously for the IPY. Two radar systems were capable of meeting this objective [Sojka et al., 2007]: the ESR located at $78^{\circ} 09^{\prime} \mathrm{N}, 16^{\circ} 12^{\prime} \mathrm{E}$, and PFISR located at $66^{\circ} 8^{\prime} \mathrm{N}, 212^{\circ} 32^{\prime} \mathrm{E}$. The positions of ESR and PFISR relative to a $\mathrm{Kp}=3$ [Hardy et al., 1987] auroral oval are shown in Figure 1. Symbols are shown every hour for the locations of the two ISRs, with + and o being PFISR and ESR respectively. The shaded region corresponds to energy fluxes in excess of $0.8 \mathrm{ergs} / \mathrm{cm}^{2} \mathrm{~s}$ from the Hardy oval. Figure 1 demonstrates why ESR is considered a polar cap location and PFISR an auroral location. Both radars were 


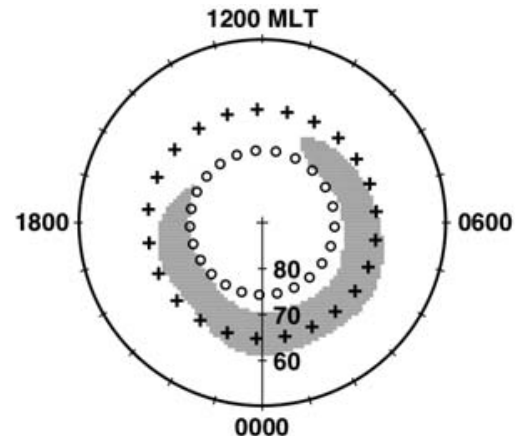

Figure 1. Locations of both PFISR $(+)$ and ESR (o) in a magnetic dipole latitude MLT polar diagram. The shaded region corresponds to auroral energy fluxes exceeding 0.8 ergs $/ \mathrm{cm}^{2}$ s from the Hardy et al. [1987] model for $\mathrm{Kp}=3$.

run for the IPY period and achieved close to $85 \%$ coverage. Both radars operated in a low duty cycle mode that produced altitude profiles of the electron density, ion temperature, electron temperature, and line-of-sight velocity along the local magnetic field line with a cadence of 10-15 minutes. The major scientific objective of this IPY campaign was to take advantage of the solar minimum conditions that would minimize the magnetospheric and solar driven disturbances while revealing dynamics in the ionosphere driven from the atmosphere below, which are expected to have tidal and planetary wave time scales. In the observed ESR and PFISR events such dynamics were indeed observed but were correlated to the geomagnetic driver - CIRs.

\section{IPY Campaign Ionospheric Observations}

[6] During the declining phase of the solar cycle and at solar minimum, CIR conditions are prevalent in the solar wind. These cause geomagnetic disturbances that last for up to a few days [McPherron and Siscoe, 2004] and are associated with $\mathrm{Kp}$ values of up to 4-5 and Dst values that reach $\sim-30 \mathrm{nT}$. These are not major disturbances; however, unlike isolated substorms that may be more intense, these CIR events are sustained for several days. Hence they provide heat input to the upper atmosphere, especially at high latitudes where convection is often enhanced. During these solar minimum conditions, the $F$-region peak was most frequently found to lie between 200 and $270 \mathrm{~km}$ [Sojka et al., 2009]. We have chosen to use $T_{i}$ observed at $\sim 300 \mathrm{~km}$ as a measure of ionospheric heating during the IPY campaign.

[7] Figure 2 shows the ESR polar region and PFISR auroral region $T_{i}$ observations at $300 \mathrm{~km}$ for 40 days during the IPY period along with the corresponding $\mathrm{Kp}$ variation. The ESR and PFISR observations contain the diurnal modulation as well as that imposed by external drivers. For this study period the solar EUV was fairly constant at an F10.7 of $\sim 70$. Although a few solar rotations during the IPY showed a modulation of F10.7, these do not provide a one-to-one correlation with the ISR $T_{i}$ heating events. The selected period is from 25 April 2007 to 4 June 2007, which corresponds to the equinox to summer transition period. The ESR had a down period of three days but otherwise the two ISR data sets include observations almost every 15 minutes.
By inspection of Figure 2 it is readily noted that the two ISRs are measuring a similar ion temperature. Heating occurs on at least two distinct time scales: short period (few hour) heating that increases $T_{i}$ at $300 \mathrm{~km}$ by many hundreds of degrees as well as a longer term (days) increase of the lowest temperature. The shorter period $T_{i}$ enhancements are probably associated with impulse heating often seen during substorms. This heating is not necessarily found simultaneously in the two ISR data sets. In contrast, the longer term heating on the order of $100 \mathrm{~K}$ is found in both the PFISR and ESR observations and is of primary interest in this study. Indeed, these enhancements in temperature correspond to times when the Kp index increases to above 2 and reaches almost 5. Each event rises in about half-a-day, and recovers more slowly over 2 to 3 days. The duration of the $\mathrm{Kp}$ events is from 1.5 to 3 days in this forty-day period. In the next section it will be shown that these events correlate directly with CIR events.

[8] The connection to the CIR fast stream is shown in Figure 3 where for the 7 May 2007 event the ISR $T_{i}, \mathrm{Kp}$, and solar wind velocity is shown over an eight-day period centered on the CIR epoch (dashed vertical line). All four panels indicate "activity" increasing at least half-a-day prior to the epoch time (a community standard described in the next section). Within the CIR, each ISR sees different heating impulses but both observe an overall heating.

\section{CIR Correlation Results}

[9] Stream interfaces during the IPY interval were determined using solar wind data from the ACE spacecraft. The data are propagated to the Earth in GSE coordinates using the modified minimum variance technique [Weimer et al., 2003; Bargatze et al., 2005]. An interface is defined as the zero crossing of E-W flow as it reverses from westward to eastward, usually following a sector boundary. This occurs
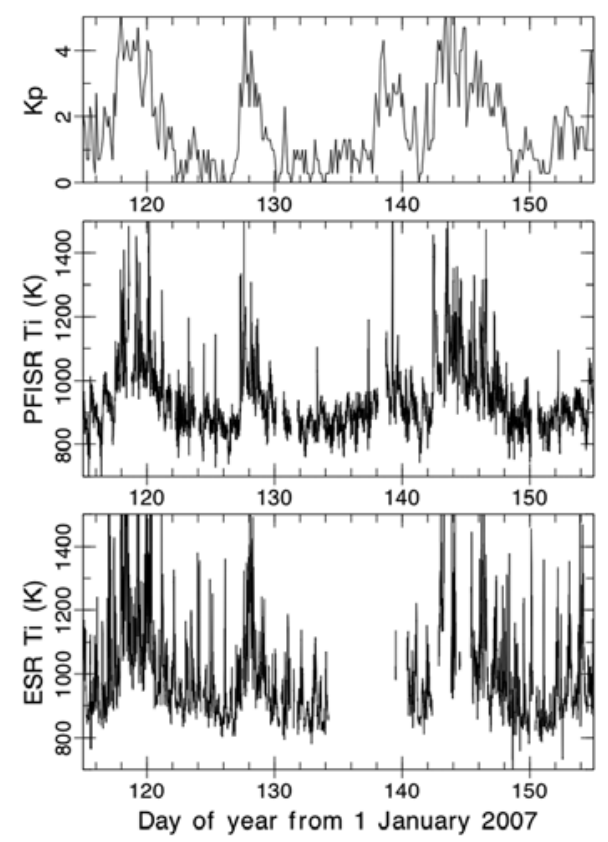

Figure 2. (bottom) ESR and (middle) PFISR $T_{i}$ at $300 \mathrm{~km}$ observations from day 115 to 155,2007 and (top) the Kp three hourly index over the same period. 


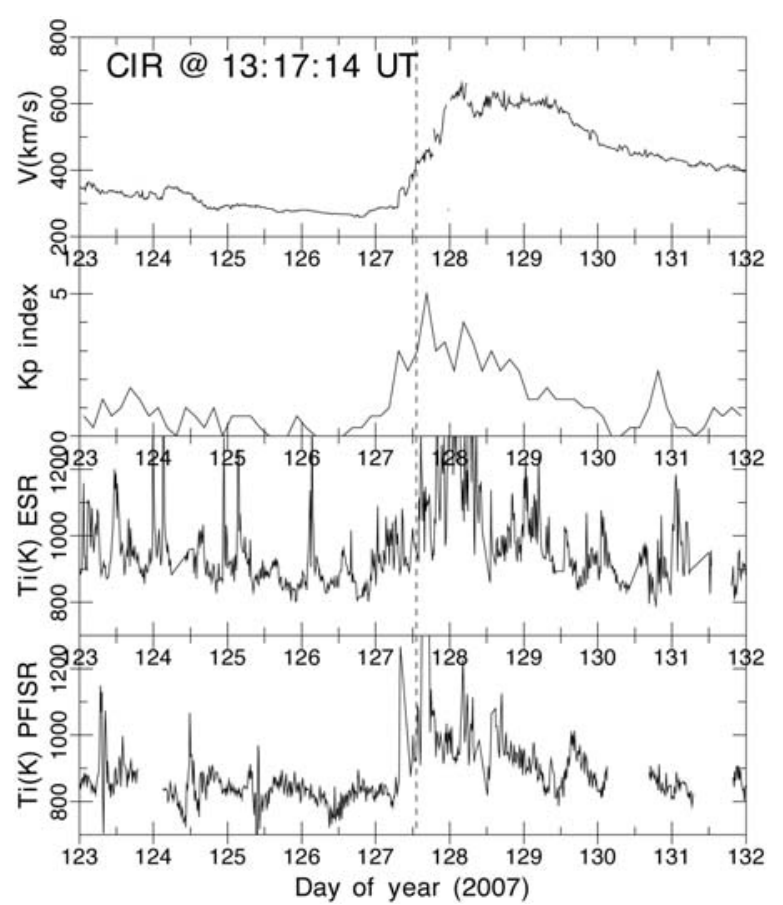

Figure 3. (bottom to top) The PFISR $T_{i}$, ESR $T_{i}, \mathrm{Kp}$ and solar wind speed for a CIR on 7 May 2007. A vertical dashed line at 1317 UT on 7 May is the CIR epoch time.

just as the density decreases sharply and the field magnitude peaks. These times then represent the CIR epoch times and enable an analysis to be performed to determine how the ionospheric temperature events observed by ESR and PFISR correlate with them. Also the ESR and PFISR data series are handled separately due to different ISR off-line periods. Hence, not all 55 CIR events are seen by each ISR.

[10] Ionospheric temperature data for each of the 55 stream interfaces were placed in rows of an ensemble matrix. Fifteen-minute samples for four days before and after the interface were used. An analysis window of width 15 samples was stepped across the ensemble matrix and a cumulative probability distribution function (cdf) was constructed. This distribution was interpolated to a uniform grid and stored in a 2-D array with universal time as the horizontal axis, the value of the temperature as ordinate, and the cumulative probability as the dependent variable. The array was contoured at deciles of the cdf to illustrate the behavior of the variable as a function of epoch time, see Figure 4 (top). Heavy lines show the three quartiles of the distribution. In Figure 4 (top) the cdf is plotted for the ESR $T_{i}$ observations at $300 \mathrm{~km}$ for the IPY year. All of the decile traces have the same correlated morphology. The ion temperature increases from a minimum to a maximum value in the day preceding the CIR epoch; maximizes in the 12 hours following the crossing; and decreases more slowly over a 2 to 4 day period. The epoch range is from -4 to 4 days. This is consistent with the 5 to 8 day recurrence rate of CIR in the IPY period. A potentially significant additional finding is that the temperature is coolest in the one day before the rapid increase. This is seen in Figure 4 (top) by all the decile lines having a distinct minimum between -1 and -2 epoch time. The increase in temperature in the rapid rise ranges from $150 \mathrm{~K}$ for the lowest decile to almost $450 \mathrm{~K}$ for the highest decile. In the context of their respective cold pre-CIR temperature, these increases correspond to $25 \%$ and $42 \%$ respectively. A similar result has been found by Borovsky and Steinberg [2006].

[11] The PFISR $T_{i}$-CIR cdf is shown in Figure 4 (bottom) in the same format as Figure 4 (top). The correlation and synchronization to epoch zero is the same as found for the ESR observations. Both the rapid heating and slower cooling may be occurring faster in this PFISR cdf plot than in the ESR cdf plot (Figure 4, top). Prior to the rapid heating there is again a distinct coldest period lasting about one day. However, the relative temperature increases are somewhat smaller; the lowest decile has a $75 \mathrm{~K}(10.7 \%)$ increase and the highest decile has a $250 \mathrm{~K}(27 \%)$ increase.

[12] McPherron and Siscoe [2009] have carried out the same type of cdf analysis between CIR events and geomagnetic indices. These include the Ap, AL, AE, Dst, SymH, and $\mathrm{PC}$ indices. For all indices the correlation found in this study using the ISR $T_{i}$ measurements are the same.

\section{Discussion and Conclusion}

[13] All IPY CIR events show a one-to-one correlation with an ionospheric heating event in the northern polar
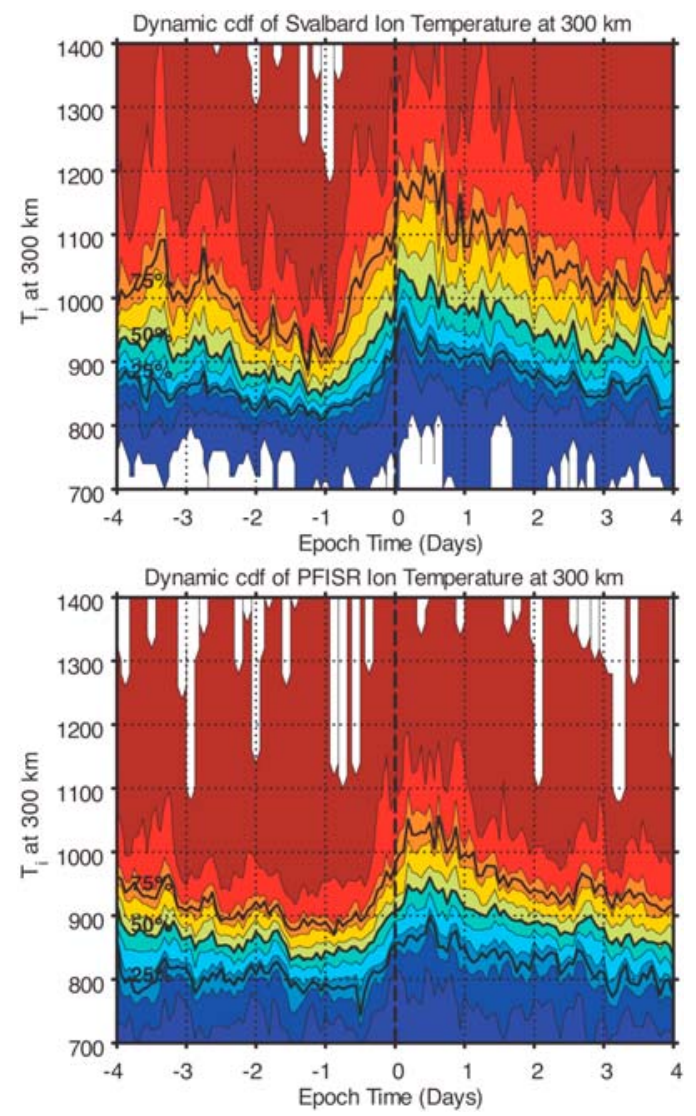

Figure 4. (top) The ESR $T_{i}$ CIR dynamic cumulative distribution function for the entire IPY period, 1 March 2007 to 29 February 2008. Thin lines separating colors, represent deciles while the three thick lines represent quartiles of the cdf. (bottom) The PFISR $T_{i}$-CIR cdf for the entire IPY period, 1 March 2007 to 29 February 2008. Thin lines, separating colors, represent deciles while the three tick lines represent quartiles of the cdf. 
region (ESR observations) and in the northern auroral region (PFISR observations). The ion temperature correlation found in Figure 2 with the geomagnetic $\mathrm{Kp}$ index provides the linkage between the solar wind, magnetosphere, and ionosphere in that it is an accepted planetary measure of geomagnetic activity generated in the magnetosphere. ESR and PFISR are located not only in different regions, but also almost $180^{\circ}$ in longitude, yet see the same overall response to each CIR, implying that they are monitoring a relatively uniform high-latitude heating mechanism. That this temporal synchronization is found for all events over one year also implies that this is the dominant system driver. In other words, CIR events drive the magnetosphere which in turn drives the ionosphere without significant delay. As Thayer et al. [2008] suggested, this driven system may well have predictable space weather relevance. The IPY PFISR-ESR observations provide the fiduciary data set to test modeling of this direct process in that the ionospheric heating is measured continuously over the multiple-day CIR events.

[14] The results presented here do not contain direct information about the thermospheric events described in the introduction. Issues of how well an ion temperature measurement made just above the $F$-region peak is indicative of the thermospheric heating needs to be investigated. The distinction between joule heating and ion frictional heating most certainly are key to understanding these auroral and polar cap observations. For example in Figure 2 , the ion temperature heating events occur on short and long periods. Hence, the argument could well be made that their relationship to the thermospheric thermal state would be quite different. It is likely that the longer period heating, with a duration of days, will best relate to the thermospheric thermal state during the CIR events. In addition, there may be additional information in the ionospheric layer motion that may better represent ionospheric/thermospheric heating during disturbed time periods.

[15] The longer term $T_{i}$ heating characteristic provides a link to the recent discoveries of thermospheric statistical periodicities that have also been found in geomagnetic indices (Kp and Ap) and CIR events [Mlynczak et al., 2008; Lei et al., 2008; Thayer et al., 2008]. The heating of the ionosphere at $300 \mathrm{~km}$ shown in Figure 2 indicates that the thermosphere has been heated. Heating the thermosphere provides the required driver for the enhanced radiated power of $\mathrm{CO}_{2}$ and $\mathrm{NO}$ observed by TIMED and enhanced drag by CHAMP. The ISR observations provide quantitative measures of the ionospheric heating as each CIR impacts the geospace system. Hence, follow on IPY studies using first principles modeling of the solar windmagnetosphere-ionosphere-thermosphere system will have ISRs providing the ground-truth of the energy flow in the ionosphere. With this system-level approach the models will test the relative coupling processes between the ionosphere and thermosphere since independent observations of the driver (CIRs) and the ionosphere and thermosphere responses are available. Follow on studies will determine the efficiency of energy flow into the ionosphere via application of the Russell-McPherron effect on the IPY ACE-ISR data sets.
[16] Acknowledgments. This research was supported by NSF grant ATM-0408592 to USU and NASA grant NNX-07AG16G to UCLA. EISCAT is an international association supported by research organizations in China (CRIRP), Finland (SA), France (CNRS, until end 2006), Germany (DFG), Japan (NIPR and STEL), Norway (NFR), Sweden (VR), and the United Kingdom (PPARC). PFISR is operated under NSF cooperative agreement ATM-0608577 to SRI International. The solar wind CIR data was obtained from the ACE and Wind satellites. The International Space Science Institute (ISSI) is gratefully acknowledged for its role in sponsoring IPY team meetings on this research topic.

\section{References}

Bargatze, L. F., R. L. McPherron, J. Minamora, and D. Weimer (2005), A new interpretation of Weimer et al.'s solar wind propagation delay technique, J. Geophys. Res., 110, A07105, doi:10.1029/2004JA010902.

Borovsky, J. E., and M. H. Denton (2006), Differences between CMEdriven storms and CIR-driven storms, J. Geophys. Res., 111, A07S08, doi:10.1029/2005JA011447.

Borovsky, J. E., and J. T. Steinberg (2006), The "calm before the storm" in $\mathrm{CIR} /$ magnetosphere interactions: Occurrence statistics, solar wind statistics, and magnetospheric preconditioning, J. Geophys. Res., 111, A07S10, doi:10.1029/2005JA011397.

Crowley, G., A. Reynolds, J. P. Thayer, J. Lei, L. J. Paxton, A. B. Christensen, Y. Zhang, R. R. Meier, and D. J. Strickland (2008), Periodic modulations in thermospheric composition by solar wind high speed streams, Geophys. Res. Lett., 35, L21106, doi:10.1029/2008GL035745.

Hardy, D. A., M. S. Gussenhoven, R. Raistrick, and W. J. McNeil (1987), Statistical and functional representations of the pattern of auroral energy flux, number flux, and conductivity, J. Geophys. Res., 92, 12,275$12,294$.

Lei, J., J. P. Thayer, J. M. Forbes, E. K. Sutton, and R. S. Nerem (2008), Rotating solar coronal holes and periodic modulation of the upper atmosphere, Geophys. Res. Lett., 35, L10109, doi:10.1029/2008GL033875.

McPherron, R. L., and G. Siscoe (2004), Probabilistic forecasting of geomagnetic indices using solar wind air mass analysis, Space Weather, 2, S01001, doi:10.1029/2003SW000003.

McPherron, R. L., and G. Siscoe (2009), Role of the Russell-McPherron effect in the acceleration of relativistic electrons, J. Atmos. Sol. Terr. Phys., 71, 1032-1044, doi:10.1016/j.jastp.2008.11.002.

McPherron, R. L., and J. Weygand (2006), The solar wind and geomagnetic activity as a function of time relative to corotating interaction regions, in Recurrent Magnetic Storms: Corotating Solar Wind Streams, Geophys. Monogr. Ser., vol. 167, edited by B. Tsurutani et al., pp. 125-138, AGU, Washington, D. C.

Mlynczak, M. G., F. J. Martin-Torres, C. J. Mertens, B. T. Marshall, R. E. Thompson, J. U. Kozyra, E. E. Remsberg, L. L. Gordley, J. M. Russell III, and T. Woods (2008), Solar-terrestrial coupling evidenced by periodic behavior in geomagnetic indexes and the infrared energy budget of the thermosphere, Geophys. Res. Lett., 35, L05808, doi:10.1029/ 2007 GL032620.

Sojka, J., R. Schunk, T. van Eyken, J. Kelly, C. Heinselman, and M. McCready (2007), Ionospheric challenges of the International Polar Year, Eos Trans. $A G U, 88(15), 771$, doi:10.1029/2007EO150003.

Sojka, J. J., M. J. Nicolls, C. J. Heinselman, and J. D. Kelly (2009), The PFISR IPY observations of ionospheric climate and weather, J. Atmos. Sol. Terr. Phys., 71, 771-785, doi:10.1016/j.jastp.2009.01.001.

Temmer, M., B. Vršnak, and A. M. Veronig (2007), Periodic appearance of coronal holes and the related variation of solar wind parameters, Sol. Phys., 241, 371-383, doi:10.1007/s11207-007-0336-1.

Thayer, J. P., J. Lei, J. M. Forbes, E. K. Sutton, and R. S. Nerem (2008), Thermospheric density oscillations due to periodic solar wind high-speed streams, J. Geophys. Res., 113, A06307, doi:10.1029/2008JA013190.

Weimer, D. R., D. M. Ober, N. C. Maynard, M. R. Collier, D. J. McComas, N. F. Ness, C. W. Smith, and J. Watermann (2003), Predicting interplanetary magnetic field (IMF) propagation delay times using the minimum variance technique, J. Geophys. Res., 108(A1), 1026, doi:10.1029/ 2002JA009405.

C. J. Heinselman, J. D. Kelly, M. J. Nicolls, and A. P. van Eyken, Center for Geospace Studies, SRI International, 333 Ravenswood Avenue, Menlo Park, CA 94025, USA. (michael.nicolls@sri.com)

R. L. McPherron, Institute of Geophysics and Planetary Physics, University of California, Los Angeles, CA 90095, USA. (mcpherron@igpp.ucla.edu)

J. J. Sojka, Center for Atmospheric and Space Sciences, Utah State University, Logan, UT 84322-4405, USA. (jan.sojka@usu.edu) 